\title{
15. On the Buckling under Edge Thrusts of a Rectangular Plate Clamped at Four Edges.
}

\author{
By Katsutada SEZAWA.
}

Aeronautical Research Institute, Tokyo Imperial University.

(Rec. Jan. 31, 1931. Comm. by K. Suyehiro, M.I.A., Feb. 12, 1931.)

So long ago as 1891 Bryan $^{1)}$ obtained the solution of the problem of a rectangular plate which is supported at four edges and subject to edge thrusts, while a similar problem in which a pair of opposite edges is clamped and the other is supported was later dealt with by Reissner.") In spite of its importance in structural constructions, the case, where the differential equation of equilibrium of a plate is fulfilled and four edges of the same plate are clamped, has not, as far as I am aware, yet been solved. I have, however, found that such a case may be solved with a small modification on the boundary conditions: this modification does not much affect the result of solution of an elastic problem concerning a rectangular plate. ${ }^{3)}$

We shall take a two-dimensional problem in which the axes of $x$ and $y$ are directed parallel to edges, their origin being taken at the centre of the plate. Let $w$ be the deflection of the plate and $P_{1}, P_{2}$ be the thrusts per unit breadth of the plate acting parallel to $x$ - and $y$-axes respectively, while $D_{1}$ is defined by

$$
D_{1}=\frac{1}{12} \frac{E h^{3}}{\left(1-\sigma^{2}\right)},
$$

where $E$ is Young's modulus, $\sigma$ Poisson's ratio and $h$ the thickness of the plate. The equation of motion of a plate at critical stability is then expressed by

$$
D_{1}\left(\frac{\partial^{4} w}{\partial x^{4}}+2 \frac{\partial^{4} w}{\partial x^{2} \partial y^{2}}+\frac{\partial^{4} w}{\partial y^{4}}\right)+P_{1} \frac{\partial^{2} w}{\partial x^{2}}+P_{2} \frac{\partial^{2} w}{\partial y^{2}}=0
$$

When four edges of the plate are clamped, we have

$$
x= \pm \frac{a}{2} ; \quad w=0, \quad \frac{\partial w}{\partial x}=0 \text {, }
$$

1) G. H. Bryan: Proc. Math. Soc., London, 22 (1891), 54-67.

2) H. Reissner: Zentralblatt d. Bauverwaltung, 29 (1909), 94-96.

3) K. Sezawa: Engineering, London, 116 (1923), 188-191. 
No. 2.] On the Buckling under edge Thrusts of a Rectangular Plate Clamped.

$$
y= \pm \frac{b}{2} ; \quad w=0, \quad \frac{\partial w}{\partial y}=0,
$$

where $a$ and $b$ are the length and the breadth of the plate. Writing in (2)

$$
\left.\left.\left.w=W_{1} \frac{\cos }{\sin }\right\} \frac{m \pi x}{a}+W_{2} \frac{\cos }{\sin }\right\}\right\} \frac{n \pi y}{b}
$$

where $W_{1}$ and $W_{2}$ are functions of $y$ and $x$ respectively, $m$ and $n=1,3,5, \ldots \ldots$ for $\cos \frac{m \pi x}{a}$ and $\cos \frac{n \pi y}{b}$, and $m$ and $n=2,4,6, \ldots \ldots$ for $\sin \frac{m \pi x}{a}$ and $\sin \frac{n \pi y}{b}$ ( $m$ and $n$ in the latter case will be written $m^{\prime}$ and $n^{\prime}$ hereafter), we obtain

$$
\begin{gathered}
{\left[\frac{d^{4} W_{2}}{d x^{4}}+\left\{\frac{P_{1}}{D_{1}}-2\left(\frac{n \pi}{b}\right)^{2}\right\} \frac{d^{2} W_{2}}{d x^{2}}-\left(\frac{n \pi}{b}\right)^{2}\left\{\frac{P_{2}}{D_{1}}-\left(\frac{n \pi}{b}\right)^{2}\right\} W_{2}\right] \cos \frac{n \pi y}{b}} \\
+\left[\frac{d^{4} W_{1}}{d y^{4}}+\left\{\frac{P_{2}}{D_{1}}-2\left(\frac{m \pi}{a}\right)^{2}\right\} \frac{d^{2} W_{1}}{d y^{2}}-\left(\frac{m \pi}{a}\right)^{2}\left\{\frac{P_{1}}{D_{1}}\right.\right. \\
\left.\left.-\left(\frac{m \pi}{a}\right)^{2}\right\} W_{1}\right] \cos \frac{m \pi x}{a}=0
\end{gathered}
$$

This is merely a representative expression and we may take $m^{\prime}$ in lieu of $m$ and similarly for $n^{\prime}$. The equation (6) may be satisfied by putting each of the expressions contained within square brackets to zero. Solving two differential equations thus formed, we find the general solution in the following form:

$$
\begin{aligned}
& \left.\left.\left.w=\left[A \begin{array}{c}
\cos \\
\sin
\end{array}\right\} \frac{\sqrt{\sqrt{a^{2}+\beta^{2}}-a}}{b} x+B \begin{array}{c}
\cosh \\
\sinh
\end{array}\right\} \frac{\sqrt{\sqrt{a^{2}+\beta^{2}}+\alpha}}{b} x\right]_{\sin }^{\cos }\right\} \frac{m \pi y}{a} \\
& \left.\left.\left.\left.+\left[C_{\sin }^{\cos }\right\} \frac{\sqrt{\sqrt{\gamma^{2}+\delta^{2}}-\gamma}}{a} y+D D_{\sinh }^{\cosh }\right\} \frac{\sqrt{\sqrt{r^{2}+\delta^{2}}+\gamma}}{a} y\right]\right]_{\sin }^{\cos }\right\} \frac{m \pi x}{a},
\end{aligned}
$$

where

$$
\begin{array}{ll}
-\alpha=\frac{P_{1} b^{2}}{2 D_{1}}-n^{2} \pi^{2}, & -\gamma=\frac{P_{2} a^{2}}{2 D_{1}}-m^{2} \pi^{2}, \\
\beta^{2}=n^{2} \pi^{2}\left(\frac{P_{2} b^{2}}{D_{1}}-n^{2} \pi^{2}\right), & \delta^{2}=m^{2} \pi^{2}\left(\frac{P_{1} a^{2}}{D_{1}}-m^{2} \pi^{2}\right) .
\end{array}
$$

Now it is exceedingly difficult to make these expressions to satisfy at the same time all the boundary conditions, so that I have made the following approximation: the deflection at the edges is zero, but 
the slope at the edges is not everywhere zero except at the middle points of all the edges, when $m$ and $n=1,3,5, \ldots \ldots$, besides at the corners. When $m$ and $n=2,4,6, \ldots \ldots$, the slope is accurately zero at $x / a$ and $y / b=1 / 4,1 / 8,1 / 12, \ldots \ldots$. By applying the above basis of criterion and taking merely the case of odd $n$, we find

$$
\begin{aligned}
& \left.A \underset{\sin }{\cos }\} \frac{\sqrt{\sqrt{\alpha^{2}+\beta^{2}}-\alpha}}{2 b} a+B \begin{array}{c}
\cosh \\
\sinh
\end{array}\right\} \frac{\sqrt{\sqrt{a^{2}+\beta^{2}}+\alpha}}{2 b} a=0, \\
& \mp\left\{A \frac{\sqrt{a^{2}+\beta^{2}-\alpha}}{b} \sin \right\} \frac{\sqrt{\sqrt{a^{2}+\beta^{2}}-\alpha}}{2 b} a
\end{aligned}
$$

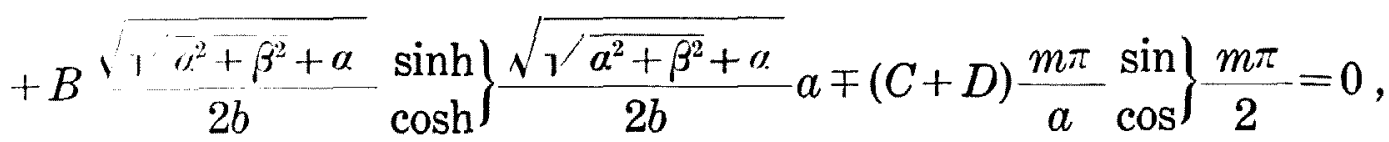

$$
\begin{aligned}
& \begin{array}{c}
2 b \quad \cosh \frac{2 b}{\sqrt{\sqrt{\gamma^{2}+\delta^{2}}-\gamma}} \\
2 a
\end{array} b+D \cosh \frac{\sqrt{\sqrt{\gamma^{2}+\delta^{2}}+\gamma}}{2 a} b=0, \\
& -\left(A \sin \frac{\sqrt{\sqrt{a^{2}+\beta^{2}}-\alpha}}{2 m b} a+B \sinh \frac{-(A+B)}{2 m b}\right\} \frac{n \pi}{b} \sin \frac{n \pi}{2} \\
& =C \frac{\sqrt{\sqrt{\gamma^{2}+\delta^{2}}-\gamma}}{a} \sin \frac{\sqrt{\sqrt{\gamma^{2}+\delta^{2}}-\gamma}}{2 a} b \\
& -D \frac{\sqrt{\sqrt{\gamma^{2}+\delta^{2}}+\gamma}}{a} \sinh \frac{\sqrt{\sqrt{\gamma^{2}+\delta^{2}+\gamma}}}{2 a} b
\end{aligned}
$$

the upper and lower terms of the first and third members of (10) and also of the left-hand member of (12) being taken according as $m$ is odd or even.

Eliminating $A, B, C, D$ between (9), (10), (11), (12) and applying the relations in (8), we may find the critical values of the thrusts $P_{1}$ and $P_{2}$. As it is almost impossible to obtain the critical thrusts in such a manner, I have employed a method of trial and error by which, assuming different values of $P_{1}$ and $P_{2}$, only those values of $P_{1}$ and $P_{2}$ which satisfy accurately all the relations (8), (9), (10), (11), (12) are picked up.

The result of the culculation shows that, in the case of a square plate, whose sides are of a length $a$, the following sets of $P_{1}$ and $P_{2}$ are critical thrusts acting at the edges. 
No. 2.] On the Buckling under edge Thrusts of a Rectangular Plate Clamped. 51

\begin{tabular}{|c|c|c|c|c|c|c|c|c|c|c|c|}
\hline$P_{2} / P_{1}$ & 0 & $1 / 4$ & $1 / 3$ & $1 / 2$ & $3 / 4$ & 1 & $-1 / 4$ & $-1 / 2$ & -1 & $-3 / 2$ & -2 \\
\hline$\frac{P_{1} a^{2}}{D_{1} \pi^{2}}$ & 10.65 & 8.80 & 8.40 & 7.48 & 6.41 & 5.61 & 13.12 & 15.20 & 17.4 & 18.75 & 20.0 \\
\hline$\frac{P_{2} a^{2}}{D_{1} \pi^{2}}$ & 0 & 2.20 & 2.80 & 3.74 & 4.81 & 5.61 & -3.28 & -7.60 & -17.4 & -28.1 & -40.0 \\
\hline
\end{tabular}

The case, in which the ratio of the length $a$ to the breadth $b$ of a rectangular plate is not unity and the thrust $P_{2}$ is zero, is of a great importance in connection with the problem of engineering and I have also evaluated the value of $P_{1}$ for a certain range of $a / b$, the result being shown in the following table:

$$
\frac{P_{1} \alpha^{2}}{D_{1^{2}} \pi^{2}}
$$

\begin{tabular}{|c|c|c|c|c|c|}
\hline$a^{\prime} b$ & 0 & $1 / 2$ & 1 & $3 / 2$ & 2 \\
\hline & 4.00 & 5.14 & 10.65 & 30.0 & \\
\hline Number of & & & 11.8 & 18.4 & 27.7 \\
\hline length & & & & 20.6 & 24.2 \\
\hline & & & & & 36.5 \\
\hline
\end{tabular}

Evidently the buckling of a plate takes place at the smallest value of critical thrusts corresponding to various types of corrugated forms and I have shown such cases with underlined figures.

The problem of the buckling under edge thrusts of a long elastic plate clamped at periphery has already been studied by Nishimura ${ }^{1)}$ and me. It was found that the critical thrust acting parallel to the long edge is approximately equal to $7.06 D_{1} \pi^{2} / b^{2}$ and the half wave length of the bulge is nearly $0.63 b$. The present investigation of the stability of a plate of a finite length tells us that in the case of $a^{\prime} b=3 / 2$ the critical thrust is equal to $8.17 D_{1^{2}} \pi^{2} / b^{2}\left(=18.4 D_{1^{2}} / a^{2}\right)$ and the half wave length about $0.75 b$ and in the case of $a / b=2$ the critical thrust is of the magnitude $6.05 D_{1} \pi^{2} / b^{2}\left(=24.2 D_{1} \pi^{2} / a^{2}\right)$ and the half wave length about $0.67 b$. It will, therefore, be seen that the condition of the buckling in the neighbourhood of $a / b=2$ is practically the same what it would be for $a / b=\infty$.

The details as well as the extension of the present problem will be published in the Report of the Aeronautical Research Institute.

1) K. Sezawa and G. Nishimura: Jour. Soc. Nav. Arch., Tokyo, 47 (1931), which is now under press. 